\title{
Peripheral administration of tau aggregates triggers intracerebral tauopathy in transgenic mice
}

\author{
Florence Clavaguera • Jürgen Hench • Isabelle Lavenir • \\ Gabriel Schweighauser · Stephan Frank • \\ Michel Goedert · Markus Tolnay
}

Received: 28 November 2013 / Revised: 8 December 2013 / Accepted: 9 December 2013 / Published online: 21 December 2013

(C) Springer-Verlag Berlin Heidelberg 2013

Soluble tau forms insoluble aggregates following the intracerebral injection of tau inclusions [3, 4]. This is reminiscent of prions, the intracerebral injection of which induces aggregation of cellular prion protein $\left(\mathrm{PrP}^{\mathrm{C}}\right)$ [9]. Multiple routes of administration can transmit prion diseases, with the intracerebral route being more effective than the intraperitoneal route, which is in turn more effective than the oral route [7]. The oral administration of aggregated apolipoprotein A-II [11] and aggregated amyloid protein A (AA) [8] has also been shown to promote systemic amyloidosis. Moreover, intraperitoneally injected aggregated $\mathrm{A} \beta$-containing extracts increased cerebral $\beta$-amyloidosis [5]. Here, we report for the first time that the intraperitoneal injection of tau seeds can also induce intracerebral tauopathy.

We used homozygous and heterozygous mice transgenic for human mutant P301S tau [2]. In heterozygous mice, tau aggregation forms later than in homozygous mice, and heterozygous mice live longer than their homozygous counterparts (15 months as opposed to 6 months).

M. Goedert and M. Tolnay contributed equally to this work.

Electronic supplementary material The online version of this article (doi:10.1007/s00401-013-1231-5) contains supplementary material, which is available to authorized users.

F. Clavaguera $\cdot$ J. Hench $\cdot$ G. Schweighauser $\cdot$ S. Frank .

M. Tolnay $(\triangle)$

Department of Neuropathology, Institute of Pathology, University

Hospital, 4031 Basel, Switzerland

e-mail: Markus.Tolnay@usb.ch

I. Lavenir · M. Goedert $(\square)$

Medical Research Council Laboratory of Molecular Biology,

Cambridge CB2 0QH, UK

e-mail:mg@mrc-lmb.cam.ac.uk
Brainstem extracts from 6-month-old homozygous P301S tau transgenic mice (prepared and analysed as previously described [3]) were injected into the peritoneal cavity of 3-month-old heterozygous mice, an age at which they lack tau deposits. The mice were analysed 9 months after the final injection.

The number of Gallyas silver-positive cells was assessed using a scanning light microscope. Maps of entire brain sections, along with the annotation of the brain outline and artificially altered areas, were used for the counting of black Gallyas-positive and blue haematoxylin-positive structures (Supplementary Fig. 4). Gallyas per cell nucleus $(\mathrm{G} / \mathrm{N})$ ratios were counted, thus reflecting the number of silver-positive cells. $\mathrm{G} / \mathrm{N}$ ratios were calculated per field of view $(20 \times$ objective, $661.5 \times 372.1 \mu \mathrm{m})$. Gaussblurred ratiometric images (sigma $=70$ pixels, pixel edge length $=3.45 \mu \mathrm{m}$ ) were superimposed for the control and experimental groups. Subsequently, a pixel-wise Student's $t$ test was performed and the probability $(p)$ values were plotted, with the statistically significant portion $(p<0.05)$ being represented as a colour map (Fig. 2). Our ratiometric approach $(\mathrm{G} / \mathrm{N})$ only compares equivalent brain regions to each other. Thus, despite anatomical differences in densities of nuclei, our comparative maps remain unbiased.

Although groups of mice injected intraperitoneally (IP) with brainstem homogenate from either P301S tau transgenic mice (with tau filaments) or control (CO) mice (without tau filaments) formed Gallyas-positive structures in the brain, there were also significant differences between the two groups (Fig. 1). G/N ratios (Fig. 2a, left panel) showed a marked increase in the number of silver-positive structures in the brainstem and neocortex of mice IP injected with $\mathrm{P} 301 \mathrm{~S}$ brainstem extract. The probability map (Fig. 2a, right panel) showed large differences in secondary motor cortex, ventral orbital cortex and olfactory nucleus. 


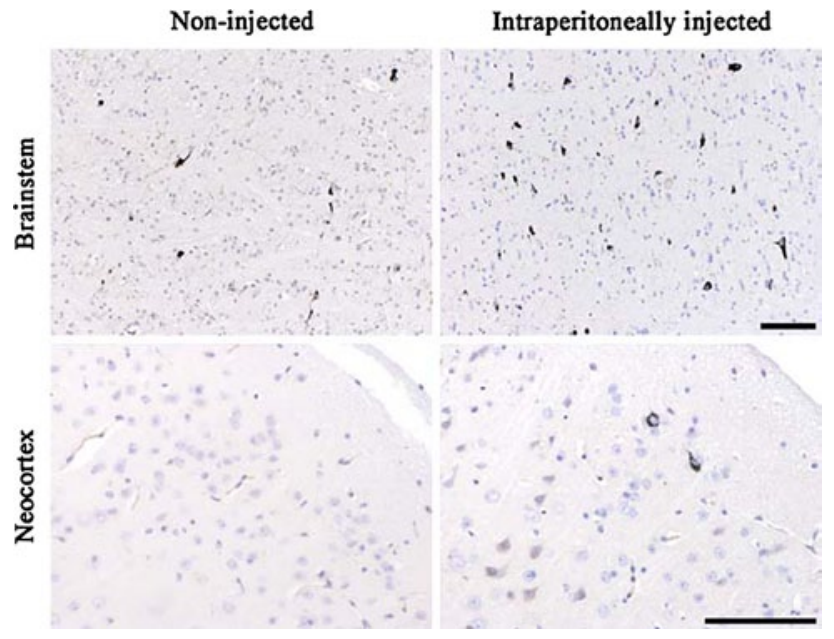

Fig. 1 Intraperitoneal injection of aggregated tau-rich homogenate increases the number of silver-positive tau inclusions in brain. Gallyas-Braak staining of the brainstem of 12-month-old mice heterozygous for transgenic human mutant P301S tau showed an increase in the number of silver-positive structures following the intraperitoneal injection of brainstem extract from 6-month-old homozygous mice, when compared with non-injected controls. The mice were injected at the age of 3 months. Silver staining was negative in the neocortex of non-injected animals, with some silver-positive structures in the neocortex of intraperitoneally injected mice. The brain sections were counterstained with haematoxylin-eosin. Scale bar $100 \mu \mathrm{m}$

More ventral regions, including pallidum and lateral preoptic area, showed more silver-positive inclusions in P301S brainstem-injected mice, as did lateral habenular nucleus, thalamic nucleus, pretectal nucleus and mesencephalic reticular formation. In the brainstem, pontine reticular nucleus, gigantocellular reticular nucleus, median vestibular nucleus and solitary tract showed the largest increase in Gallyas-positive inclusions (Supplementary Fig. 2). The hippocampus remained unaffected. At the age of analysis (12 months), brains of heterozygous P301S mice develop neuronal inclusions made of hyperphosphorylated (AT8 positive, pretangles) and aggregated tau (AT100- and Gallyas silver-positive, classical tangles). Both AT8 and AT100 tau immunoreactivity (Supplementary Fig. 3) as well as silver staining in IP-injected mice were increased compared to non-injected mice, but the pattern (brain distribution and morphology) of tau pathology remained unchanged. Age-matched wild-type mice injected intraperitoneally with P301S brainstem extract failed to develop cerebral tauopathy.

We previously showed that the intracerebral injection of silver-positive brainstem extract from mice homozygous for human mutant P301S tau into mice transgenic for human wild-type tau (line ALZ17) induced the formation of silverpositive inclusions [3]. To investigate if this is also true of another mouse model of tauopathy, we injected brainstem extract from homozygous mice transgenic for human mutant P301S tau into the hippocampus and overlying cerebral cortex of 3-month-old heterozygous mice and compared the relative efficiency of intracerebral and intraperitoneal injections after 9 months. Following intracerebral injection, numerous Gallyas-positive structures were present in the CA1 region of the hippocampus and in the secondary motor cortex (Supplementary Fig. 1A). No silver-positive structures were present in these regions following the intraperitoneal injection of control extracts. Heat maps revealed a significant increase in the $\mathrm{G} / \mathrm{N}$ ratio in the hippocampus following the intracerebral injection of P301S brainstem extract into heterozygous transgenic P301S tau mice (Fig. 2b, left panel). The p maps showed statistically significant differences between groups in the hippocampal area, which was a site of intracerebral injection (Fig. 2b, right panel; Supplementary Fig. 1B). Moreover, the G/N ratio was increased more in brainstem and neocortex following intracerebral than after intraperitoneal injections. Heart, lungs, liver, spleen and kidneys from injected heterozygous P301S tau mice were all silver-negative and tau-negative. No sign of inflammation was detected in either the brain or the periphery.

These findings demonstrate that aggregated tau seeds, such as prions and $A \beta$ aggregates, can reach the central nervous system from the periphery. The underlying mechanisms remain to be determined. The replication of peripherally applied prions and their translocation to the brain are dependent on haematopoietic and stromal immune cells, together with the sympathetic innervation of abdominal lymphoid organs [1]. In mouse models of AA-amyloidosis, peripheral blood monocytes transported the aggregates [10]. It therefore appears that amyloid seeds can be carried by blood cells. This may also be true of aggregated tau. Seeding may require the presence of unfolded monomers [6]. It remains to be determined if tau seeds are able to replicate in the peripheral nervous system. Their transport to the brain may not require endogenous tau, which is not expressed by lymphoreticular cells. This is unlike prion propagation, which depends on the expression of $\operatorname{PrP}^{\mathrm{C}}$ by stromal and haematopoietic cells. Because the central nervous system is affected in clinical tauopathy, the intracerebral route of seed delivery could be expected to be more effective than the intraperitoneal route. This was the case here, where more silverpositive structures formed in brainstem, hippocampus and neocortex following the intracerebral injection of $5 \mu 1$ brainstem homogenate $(0.19 \mu \mathrm{g}$ of $\tau / \mu \mathrm{l}$ of homogenate) than after the intraperitoneal injection of $200 \mu 1$ homogenate $(0.19 \mu \mathrm{g}$ of $\tau / \mu \mathrm{l}$ of homogenate). It follows that, like prion diseases, tauopathies can be seeded in the brain by tau aggregates delivered peripherally, although the intraperitoneal administration was less effective than the 
A

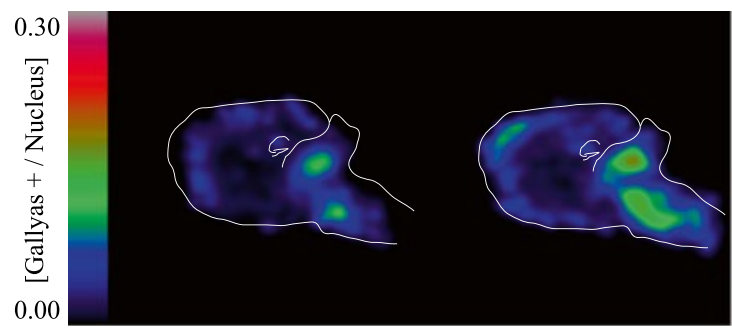

Non-injected

(CO)

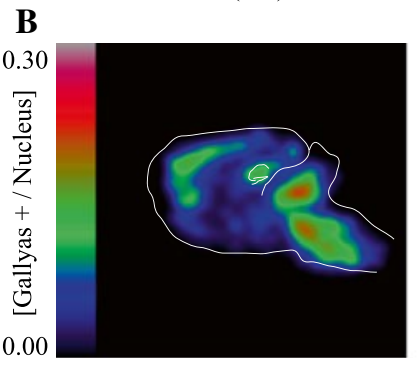

Intracerebrally injected

(IC)

(IP)

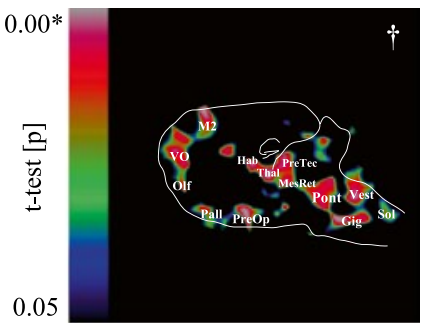

$\mathrm{CO}<$ IP

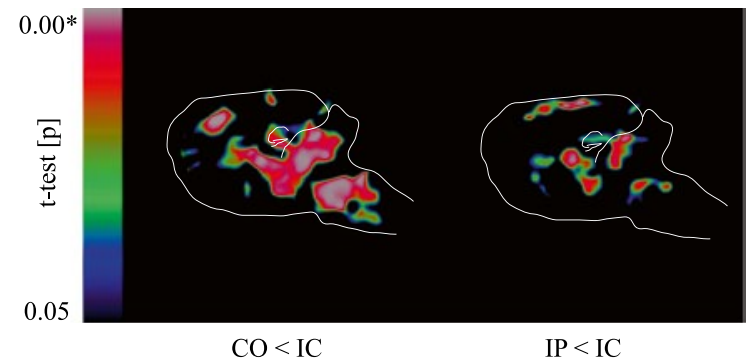

$\mathrm{CO}<\mathrm{IC}$

IP $<$ IC

Fig. 2 Gallyas silver-positive structures per cell nucleus (G/N ratios) in non-injected controls (CO) and in mice injected with aggregated tau-rich homogenate either intraperitoneally (IP) or intracerebrally (IC). a Left panel colour map representing average $\mathrm{G} / \mathrm{N}$ ratios ( $\mathrm{CO}$ and IP) of five sagittal brain sections per group. Right panel statistically significant differences were determined by pixel-based $t$ tests and colour coded in the range of $<0.05$. IP-injected mice had higher $\mathrm{G} / \mathrm{N}$ ratios in the brainstem and neocortex than $\mathrm{CO}$ mice. $M 2$ secondary motor cortex, $V O$ ventral orbital cortex, Olf olfactory nucleus, Pall pallidum, PreOp lateral preoptic area, $\mathrm{Hab}$ lateral habenular nucleus, Thal thalamic nucleus, PreTec pretectal nucleus, MesRet mesencephalic reticular formation, Pont pontine reticular nucleus, Gig gigantocellular nucleus, Vest median vestibular nucleus, Sol

intracerebral route. These findings underscore the urgent need for additional work on the aetiology and pathogenesis of tauopathies.

Acknowledgments We thank Markus Bänziger, Sabine Ipsen and Sabina Weingärtner for technical support. This work was supported by the Swiss National Science Foundation (310030_135214), the VELUX Foundation and the UK Medical Research Council (U105184291).

\section{References}

1. Aguzzi A, Heikenwalder M (2006) Pathogenesis of prion diseases: current status and future outlook. Nat Rev Microbiol 4:765-775

2. Allen B, Ingram E, Takao M et al (2002) Abundant tau filaments and nonapoptotic neurodegeneration in transgenic mice expressing human P301S tau protein. J Neurosci 22:9340-9351

3. Clavaguera F, Bolmont T, Crowther RA et al (2009) Transmission and spreading of tauopathy in transgenic mouse brain. Nat Cell Biol 11:909-913 solitary tract. Dagger symbol an enlarged version is given in Supplementary Fig. 2. Each group consisted of five mice. Asterisk rounding leads to an absolute zero, which reflects an infinitely small value ( $p$ is not identical to 0 ). b Left panel colour map representing average $\mathrm{G} / \mathrm{N}$ ratios of five sagittal brain sections from IC-injected mice. Right panel statistically significant differences between the three groups were determined by pixel-based $t$ tests and colour coded in the range of $<0.05$. Statistically significant differences were apparent between non-injected and injected animals, with the major differences between IP- and IC-injected animals being located in the hippocampus, one of the two intracerebral injection sites. Each group consisted of five mice. Asterisk rounding leads to an absolute zero, which reflects an infinitely small value ( $p$ is not identical to 0 )

4. Clavaguera F, Akatsu H, Fraser G et al (2013) Brain homogenates from human tauopathies induce tau inclusions in mouse brain. Proc Natl Acad Sci (USA) 110:9535-9540

5. Eisele YS, Obermüller U, Heilbronner G et al (2010) Peripherally applied Abeta-containing inoculates induce cerebral beta-amyloidosis. Science 330:980-982

6. Hurshman AR, White JT, Powers ET, Kelly JW (2004) Transthyretin aggregation under partially denaturing conditions is a downhill polymerization. Biochemistry 43:7365-7381

7. Kimberlin RH, Walker CA (1986) Pathogenesis of scrapie (strain $263 \mathrm{~K}$ ) in hamsters infected intracerebrally, intraperitoneally or intraocularly. J Gen Virol 67:255-263

8. Lundmark K, Westermark GT, Nyström S, Murphy CL, Solomon A, Westermark P (2002) Transmissibility of systemic amyloidosis by a prion-like mechanism. Proc Natl Acad Sci (USA) 99:6979-6984

9. Prusiner SB (1998) Prions. Proc Natl Acad Sci (USA) 95:13363-13383

10. Sponarova J, Nyström SN, Westermark GT (2008) AA-amyloidosis can be transferred by peripheral blood monocytes. PLoS One 3:e3308

11. Xing Y, Nakamura A, Chiba T et al (2001) Transmission of mouse senile amyloidosis. Lab Invest 81:493-499 